\title{
Intraexaminer and interexaminer reliability of manual palpation and pressure algometry of the lower limb nerves in asymptomatic subjects
}

\author{
Caitriona Fingleton*, Lucy Dempsey*, Keith Smart+, Catherine Doody* \\ *School of public health, physiotherapy \& population science, University College Dublin, Belfield, Dublin 4, Ireland \\ +St Vincent's University Hospital, Elm Park, Merrion Road, Dublin 4, Ireland
}

\begin{abstract}
Objectives: Nerve palpation is a method of clinically identifying mechanosensitivity of neural tissue by means of pressure algometry and manual palpation. There are few investigations of the reliability of lower limb nerve palpation, and femoral nerve palpation has never been previously reported. The aim of this study was to investigate the reliability of nerve palpation of the femoral, sciatic, tibial and common peroneal nerves and to report normative values for the femoral nerve.
\end{abstract}

Methods: The 4 lower limb nerves were palpated in 39 healthy volunteers using pressure algometry and manual digital palpation. Measurements were taken twice by 1 rater (intra-rater reliability) and once by a $2^{\text {nd }}$ rater (inter-rater reliability).

Results: Intraclass Correlation Coefficients for pressure pain thresholds (PPTs) via pressure algometry of the femoral, common peroneal, tibial and sciatic nerves were $0.69,0.84,0.64$ and 0.9 for intra-rater reliability respectively and $0.82,0.7,0.56$, and 0.75 for inter-rater reliability. Kappa values for manual palpation were $0.59,0.550 .42$ and 0.60 for intra-rater reliability and $0.30,0.49,0.37$ and 0.60 for inter-rater reliability. Males demonstrated significantly higher PPTs than females for the femoral, sciatic and tibial nerves, and differences in PPTs were present between right and left sides.

Conclusions: Nerve palpation of the femoral, common peroneal, and sciatic nerves using pressure algometry demonstrated good to excellent reliability, while the tibial nerve PPTs showed moderate to good reliability. Manual palpation measurements demonstrated fair to moderate reliability. Further investigation is warranted regarding the reliability of manual nerve palpation within a clinical population. 


\section{INTRODUCTION}

Neural tissue mechanosensitivity may be assessed by neural tissue provocation tests such as nerve palpation. Mechanical palpation using pressure algometry and manual palpation with the thumb have a high degree of clinical utility as they may be performed as part of a standard bedside examination ${ }^{1,2}$

Findings of localised and widespread hyperalgesia are suggestive of pain sensitization ${ }^{3}$. Increased sensitivity to nerve palpation has been observed in a number of chronic pain conditions for example non-specific arm pain ${ }^{4}$, low-back pain ${ }^{5}$ and work-related upper limb pain ${ }^{6}$.It has been suggested that nerve sensitivity may be explained by peripheral sensitization mechanisms ${ }^{7}$, in which neurogenic inflammation leads to the sensitization of neural mechanoreceptors (nervi-nervorum) ${ }^{8}$. In addition, central sensitization mechanisms may play a role in nerve sensitization whereby non-noxious stimuli from the nervi-nervorum are processed abnormally in the central nervous system ${ }^{9}$. There are reports of the reliability of nerve palpation in relation to nerves of the upper $\operatorname{limb} b^{10,6,1}$ and very limited data exist in relation to the reliability of lower limb nerve palpation $^{5,11}$, despite its use in a number of studies on clinical decision-making in relation to low back and leg pain disorders ${ }^{12,2,13}$

Walsh et al. ${ }^{11}$ investigated the reliability of mechanically palpating the sciatic, tibial and common peroneal nerves of the lower limb using a pressure algometer and provided normative pressure-pain threshold (PPT) values for these nerves. Walsh and Hall ${ }^{5}$ carried out digital (manual) palpation of lower limb nerves bilaterally and simultaneously in patients with low back and leg pain and rated pain or discomfort on 
the symptomatic side in relation to the symptomatic side. However, there are conflicting reports in relation to differences in PPT measurements between symptomatic and asymptomatic sides in subjects with chronic pain, with reports of no significant differences $^{3}$ as well as reports of significant differences between sides $^{14}$. A possible explanation is the presence or absence of peripheral and central sensitization of the nervous system in chronic pain states and it may therefore be important to carry out nerve palpation of right and left sides separately. In relation to the femoral nerve, no studies have investigated the reliability of femoral nerve palpation, or reported normative PPT values. The femoral nerve crosses the hip joint, supplying muscles of the anterior thigh and innervating the knee joint ${ }^{15}$, which may make it vulnerable to sensitization in patients with longstanding pain disorders of the lower limb. In addition, there have been no studies investigating the reliability of manual palpation of lower limb nerves which have reported separate right and left sided palpation.

This aim of this study was to investigate the reliability of femoral nerve palpation, using manual pressure and pressure algometry, in addition to further testing the reliability of manual palpation and pressure algometry of the sciatic, common peroneal and tibial nerves by means of alternate unilateral palpation. The study also sought to provide normative PPT data for the femoral nerve. 


\section{METHODS}

\section{$\underline{\text { Subjects }}$}

The Quality Appraisal Tool for Studies of Diagnostic Reliability (QUAREL) guidelines was used in the design of this study ${ }^{16}$. Based on previous reliability studies, a sample of 39 participants was selected for the study ${ }^{1,5,6,11}$. All subjects were students of University College Dublin (UCD) who were over 18 years, with no chronic pain or neurological disorders and no previous history of lumbar spine or lower limb pathologies. Subjects were invited via email to take part in the study and provided written informed consent prior to participation. Ethical approval was obtained from the UCD Human Research Ethics Committee.

\section{$\underline{\text { Design }}$}

\section{Procedure}

Two raters palpated the participants' femoral, sciatic, tibial and common peroneal nerves using manual pressure with the thumb in addition to pressure algometry (Figure $1 \& 2$ ). All testing was carried out in a laboratory in UCD. Both raters were Chartered Physiotherapists. Rater 1 (C.F.) had 6 months experience of nerve palpation techniques and Rater 2 (L.D.) underwent training in nerve palpation prior to the commencement of the study. Rater 1 (C.F.) tested subjects' right and left lower limbs to gather normative data. Rater 2 (L.D.) performed the assessment on subjects' left lower limb only, to determine inter-rater reliability. Rater 1 repeated the assessment on the right lower limb to establish intra-rater reliability. Randomization software was used to determine a random order for testing, i.e. to determine which Rater went first, and whether Rater 1 tested the left or right side first, in order to minimise a potential order effect ${ }^{16 .}$ 
Participants wore shorts provided by the researchers and were positioned on a plinth in supine for palpation of the femoral and common peroneal nerves, and in prone for palpation of the tibial and sciatic nerves. The femoral nerve was palpated lateral to the femoral artery inferior to the inguinal ligament; the common peroneal nerve where it passes behind the head of the fibula as it winds forwards around the neck; the tibial nerve where it bisects the popliteal fossa, lateral to the popliteal artery; and the sciatic nerve midway between the ischial tuberosity and the greater trochanter, deep to the gluteas maximus muscle ${ }^{17,18}$. At each nerve site, palpation was performed first with manual pressure, and thereafter with the pressure algometer. An initial trial was carried out at the subject's wrist to familiarise them with the testing protocol.

\section{Manual Palpation}

Digital pressure with the thumb was used to manually palpate each of the nerve sites using the standardised procedure established by the researchers, in which a similar mildto-moderate steady pressure was applied by each examiner; the rate of pressure was established prior to the commencement of the study by means of practice sessions on a blinded third party, who provided feedback on the consistency of the pressure applications; this was repeated until consistency in pressure application was reported at all nerve sites. The method described by Jepsen et al. ${ }^{6}$ in a study on asymptomatic and symptomatic subjects was used, whereby mechanical allodynia was quantified according to none, mild, moderate or severe. As very few subjects were categorised as severe, these scores were subsequently collapsed into normal or abnormal (i.e. normal $=$ none; abnormal $=$ mild, moderate or severe), as described by Jepson et al. ${ }^{6}$. 


\section{Pressure Algometry}

An electronic digital algometer (Somedic AB) was used for all test sites. The algometer consisted of a circular probe with a $2 \mathrm{~cm}^{2}$ round rubber tip at the end connected to a pressure transducer within the handle of the unit. The applied pressure was indicated on a digital display in kilopascals $(\mathrm{kPa})$. Pressure was applied at a rate of $30 \mathrm{kPa} / \mathrm{s}$ and subjects were instructed to press the button (terminating the pressure) as soon as the feeling of pressure began to change to that of pressure and pain. The application rate of $30 \mathrm{KPa} / \mathrm{s}$, as used by Graven-Nielson et al. ${ }^{14}$, Skou et al. ${ }^{19}$ and De-la-Llave-Rincon et al. ${ }^{34}$, was chosen to give participants sufficient time to respond to the stimulus, without maintaining pressure for an excessive time; which could lead to superficial tissue injury, and in addition may prevent the investigator from maintaining a constant rate of pressure $^{10}$, which is described as one of the most difficult aspects of pressure algometry $^{20}$. Three measures were obtained from each test site with a 10 second rest period between each measurement. The mean of the three measures was calculated. Raters were blinded to all measurements, which were stored in the memory of the algometer and retrieved when testing was completed.

\section{Data Analysis}

Data analysis was carried out using the Statistical Package for Social Sciences (SPSS) version 20 .

\section{Reliability}

For both manual palpation and pressure algometry, the right sided values of Rater 1 were compared the retest values of Rater 1 to establish intra-rater reliability, and the left 
sided values of Rater 1 were compared to left sided values taken by Rater 2 to obtain inter-rater reliability.

Kappa correlation coefficients were used to determine intra-rater and inter-rater reliability of the manual nerve palpation data. Standard error and 95\% CI were also calculated for each test site ${ }^{1}$. The classification system proposed by Landis and $\mathrm{Koch}^{21}$ was used to determine the reliability level of the Kappas $(\leq 0=$ poor, $0.01-0.20=$ slight, $0.21-0.40=$ fair, $0.41-0.60=$ moderate, $0.61-0.80=$ substantial and $0.81-1=$ almost perfect $)^{22,1}$

Intraclass correlation coefficients (ICCs) were calculated to assess reliability of the mechanical PPT data. A 2 way ANOVA (random effects model) was used to calculate inter-rater reliability, and a 1 way ANOVA (random effects model) was used to find intra-rater reliability. The classification system established by Shrout and Fleiss ${ }^{23}$ was used to determine the level of reliability ( $>0.75$ excellent, $0.6-0.75$ good, $0.4-0.59$ fair and $<0.4$ poor).

The standard error of measurement (SEM) was calculated to assess the amount of variability between PPT measures attributable to measurement error ${ }^{24}$. The formula used was $\mathrm{SEM}=\mathrm{S} \sqrt{ } 1-\mathrm{ICC}^{25}$, where $\mathrm{S}$ is the pooled standard deviation ${ }^{11}$.

Mean differences with $95 \%$ confidence intervals were calculated to determine the level of agreement between PPT measurements ${ }^{11}$. Bland-Altman plots were created for intra- 
rater and inter-rater reliability of the femoral nerve to examine for the presence of systematic bias and random error ${ }^{26}$.

\section{Normative PPT Data}

The means and standard deviation (SD) at each site were calculated. Paired t-tests were used to identify whether there were significant differences between right and left sides at each site and independent t-tests were used to determine whether there were any differences between the PPTs of males and females ${ }^{11}$.

\section{$\underline{\text { RESULTS }}$}

Twenty males and nineteen females between 18 and 33 years, with a mean age of 22 years $(\mathrm{SD}=4$ years) participated in the study.

\section{Reliability}

\section{Pressure Algometry}

Intraclass correlation coefficients (ICCs) of the femoral, common peroneal, tibial and sciatic nerves were $0.69,0.84,0.64$ and 0.90 respectively for intra-rater reliability, while those for inter-rater reliability were $0.82,0.7,0.56$, and 0.75 . The SEM values and $95 \%$ confidence intervals are reported in Tables 1 and 2 . These scores demonstrate good to excellent reliability for PPTs of the femoral, common peroneal and sciatic nerves, and moderate to good reliability for tibial nerve PPTs. Mean differences between PPT measures ranged from -17 to 12 indicating a good level of agreement as both measures were close to zero ${ }^{27}$. The limits of agreement for femoral nerve intra-rater and inter-rater reliability are shown in Bland Altman plots (Figure $3 \& 4$ ). For intra-rater and inter- 
rater reliability, values were evenly scattered above and below zero and approximately $95 \%$ of values were within the limits of agreement suggesting there was no significant systematic bias or random error ${ }^{26}$. The SEM values of $16-39 \mathrm{kPa}$ for pairs of PPT readings give an indication as to the differences in 2 PPT readings that may be considered a result of measurement error.

\section{Manual Palpation}

Manual palpation measurements showed Kappa scores of 0.59, 0.550 .42 and 0.60 for intra-rater reliability of the femoral, common peroneal, tibial and sciatic nerves and Kappa scores of $0.30,0.49,0.37$ and 0.60 respectively for inter-rater reliability (Table 3 \& 4). Inter-rater reliability was classified as fair for the femoral nerve and tibial nerve and moderate for the common peroneal and sciatic nerve. Intra-rater reliability was classified as moderate for all nerves ${ }^{21}$.

\section{Preliminary Normative Values}

Means (SD) for all tests sites, males and females and for left and right sides are shown in Tables 5 and 6 . There were significant differences between left and right sides for PPTs measures of the common peroneal and tibial nerves $(p=0.007, p=0.003)$, with no significant differences between sides for the femoral or sciatic nerve. Males demonstrated significantly higher PPTs than females for the femoral, sciatic and tibial nerves $(\mathrm{p}<.0001)$, and showed no difference for the common peroneal nerve on either the left or right side $(\mathrm{p}=.09, .93)$. 


\section{DISCUSSION}

This is the first study to report on reliability of femoral nerve palpation and results demonstrated good to excellent reliability for palpation via pressure algometry of the femoral nerve, in addition to the sciatic and common peroneal nerves, and moderate reliability for the tibial nerve. Manual palpation of nerve trunks demonstrated fair to moderate reliability for all measures.

For PPT measurements via pressure algometry, ICCs of the femoral, common peroneal and sciatic nerves demonstrated good to excellent reliability (ICC $=0.69-0.9)$, and moderate to good reliability for the tibial nerve (ICC $=0.56-0.64)$; these ICCs were lower than those reported in previous investigations. Walsh and Hall ${ }^{5}$ and Walsh et al. ${ }^{11}$ reported ICCs for lower limb nerve PPTs via pressure algometry which ranged from 0.83 to 0.96 and Sterling et al. ${ }^{10}$ reported ICCs of $0.92-0.97$ in a study on upper limb nerve palpation. This discrepancy may be in part due to the experience of the two raters in the current study in palpating these anatomical landmarks and the in the practice of pressure algometry. Stubhaug et al. ${ }^{28}$ suggest that hand-held pressure algometry only works well in examiners with extensive training; however this concept is disputed by other authors ${ }^{29}$.

The ICC should be considered along with the SEM to provide an estimate of measurement precision ${ }^{25}$. The SEM scores for PPT measurements in this study ranged from $16-39 \mathrm{KPa}$, indicating that changes in PPTs must be over 16-39KPa before an examiner can be confident that a clinical change has occurred ${ }^{25}$. 
In the current study, sciatic nerve PPTs showed excellent reliability according to the ICC scores $(0.75,0.9)$, but was also found to have the lowest level of measurement precision (SEM=27-39). Changes in sciatic nerve PPT scores of up to 27-39KPa may be entirely due to measurement error, which needs to be considered by examiners when interpreting the clinical importance of a change in PPT scores. Lower precision of sciatic nerve palpation may be due to the deeper anatomical location of the nerve.

It is unclear as to why the tibial nerve ICCs showed lower reliability $(0.56,0.64)$ than other nerves. One contributing factor to the lower ICC values could be the superficial position of the tibial nerve which may have made the nerve more inclined to move upon pressure application; leading to a higher level of measurement error by raters in this study. This concept is supported by the higher SEM values in this study $(23-25 \mathrm{KPa})$. ICCs for the femoral nerve $(0.69,0.82)$, which have not previously been reported, demonstrated good to excellent reliability, and were comparable to those of the common peroneal $(0.7,0.84)$ and sciatic nerve $(0.75,0.9)$. The Bland-Altman plot for femoral nerve intra-rater reliability demonstrated a small trend for differences to become greater with higher PPT measurements. This finding suggests that measurements may be less precise in the higher range of PPT values and could be an inherent feature of pressure algometry. This trend corresponds with the lower ICC (0.69) and higher SEM (26KPa) for femoral nerve intra-rater reliability compared to inter-rater reliability $(\mathrm{ICC}=0.82$; SEM=23). Despite this, both intra-rater and inter-rater reliability Bland-Altman plots for the femoral nerve showed that there was no significant level of random error or systematic bias ${ }^{26}$. 
It was found that PPTs differed between individual nerve trunks of the lower limb. Of note, the sciatic nerve had the highest PPT mean values $(233-256 \mathrm{KPa})$, followed by the femoral nerve $(158-176 \mathrm{KPa})$. The lowest PPT mean values were shared by the common peroneal and tibial nerves $(114-140 \mathrm{KPa})$. These findings were in keeping with previous reports $^{5,11}$. It is suggested that such variations in nerve PPTs are related to differences in the anatomical position and accessibility of nerve trunks ${ }^{10}$.

Significant differences between left and right-sided PPTs were found in the current study and are in contrast with previous reports ${ }^{10,11}$. It is unclear whether differences observed in this study are inherent or secondary to measurement error. However, researchers attempted to reduce the potential for measurement error due to an order effect by randomising the order of testing between sides ${ }^{16}$. The finding that males had significantly higher PPTs than females was in keeping with the literature reporting differences in pain perception between men and women ${ }^{30,31}$. Racine et al. ${ }^{31}$ in a systematic review of the literature over 10 years on sex differences in pain perception reported that females demonstrate lower pressure pain measurements than males. In a meta-analysis of sex differences in the perception of noxious experimental stimuli, Riley et al. ${ }^{32}$ reported the largest effect sizes were for pressure pain and tolerance measures. Differences in pain perception between males and females may be influenced by many factors including gonadal hormones, genetics and psychosocial factors ${ }^{30}$.

Manual digital palpation of nerve trunks was found to have fair to moderate reliability $($ Kappa $=0.30-0.60)$ while palpation via pressure algometry was found to have moderate to excellent reliability ( $\mathrm{ICC}=0.56-0.90)$. These Kappa scores differed from those in a 
previous investigation by Walsh et al. ${ }^{5}$, which demonstrated excellent reliability for manual palpation of lower limb nerves $(\mathrm{Kappa}=0.7-0.8)$. However, these discrepancies may be partially explained by methodological differences between the 2 studies. Participants in the study by Walsh and Hall ${ }^{5}$ had low back-related leg pain and in addition, examiners palpated both sides simultaneously and asked patients to compare sides; this method appears to assume normal responses in the unaffected side and does not take into account the possible presence of mechanosensitivity on the unaffected side due to secondary hyperalgesia and peripheral and central sensitization processes ${ }^{3,33}$. Higher levels of intra-rater (Kappa 0.83$)^{13}$ and lower levels of inter-rater reliability $(\text { Kappa } 0.14)^{12}$ of lower limb palpation-based neural tissue pain provocation tests in patient populations with low-back and leg pain have also been found; findings which may also be due to differences in methodology and testing procedures. Of note, Kappa scores from this study were consistent with those from a previous investigation of upper limb manual nerve palpation in another asymptomatic group ${ }^{6}(\mathrm{Kappa}=0.29-0.69)$ suggesting that this technique may have greater reliability in a clinical population, however this requires further investigation.

\section{LIMITATIONS}

One limitation of this study was that the order of manual palpation techniques and pressure algometry was not randomised which may have created an order effect; authors attempted to minimise this by randomising the order of testing between sides and between raters. A 4-point rating scale (none, mild, moderate, severe) was used in this study for manual palpation based on previous study ${ }^{6}$, and a lack of published research in relation to palpation of the femoral nerve. However very few subjects reported severe 
responses and the scale was subsequently dichotomised in the reliability analysis ${ }^{6}$. The use of a dicotomous scale recording responses as normal or abnormal from the outset would have simplified the study design. Additionally, the limited experience of raters in this study in the practice of pressure algometry could have affected the level of measurement error in this study, though this concept has been disputed by other authors $^{29}$. Accurate palpation of nerve sites, particularly in relation to the sciatic nerve which lies deep to other sensitive structures, is another limitation of the study. Raters attempted to ensure accuracy of palpation by carefully locating nerve sites in relation to standardised anatomical landmarks ${ }^{17,18}$.

\section{CONCLUSION}

In this study, nerve palpation using pressure algometry showed a good to excellent level of reliability for the femoral, sciatic and common peroneal nerves. Further investigation of the reliability of nerve palpation of the femoral nerve, in addition to the tibial nerve, is warranted given the limited empirical study to date which has shown some discrepancies between findings. In particular, future studies could investigate the reliability of manual nerve palpation in both normal and clinical populations by means of separate right and left sided palpation. Further study of manual palpation is pertinent as the technique requires no equipment making it accessible to clinicians.

\section{ACKNOWLEDGEMENTS}

This project was funded by an Irish Research Council Embark Postgraduate Scholarship. The authors would like to thank Dr. Ricardo Segurado for help with statistical analysis and students from UCD who participated in this study. 


\section{REFERENCES}

1. Schmid AB, Brunner F, Luomajoki H, Held U, Bachmann LM, Kunzer S, et al. Reliability of clinical tests to evaluate nerve function and mechanosensitivity of the upper limb peripheral nervous system. BMC musculoskelet disord. 2009;10:11. 2. Schäfer A, Hall TM, Lüdtke K, Mallwitz J, Briffa NK. Interrater reliability of a new classification system for patients with neural low back-related leg pain. Journal of manual and manipulative therapy. 2009;17(2):109.

3. Imamura M, Imamura ST, Kaziyama HH, Targino RA, Hsing WT, De Souza LPM, et al. Impact of nervous system hyperalgesia on pain, disability, and quality of life in patients with knee osteoarthritis: a controlled analysis. Arthritis care res. 2008;59(10):1424-31.

4. Moloney N, Hall T, Doody C. An investigation of somatosensory profiles in work related upper limb disorders: a case-control observational study protocol. BMC musculoskelet disord. 2010;11(1):22.

5. Walsh J, Hall T. Reliability, validity and diagnostic accuracy of palpation of the sciatic, tibial and common peroneal nerves in the examination of low back related leg pain. Man ther. 2009;14(6):623-9.

6. Jepsen JR, Laursen LH, Hagert C-G, Kreiner S, Larsen AI. Diagnostic accuracy of the neurological upper limb examination I: inter-rater reproducibility of selected findings and patterns. BMC neurol. 2006;6(1):8.

7. Hall T, Elvey R. Nerve trunk pain: physical diagnosis and treatment. Man ther. 1999;4(2):63-73.

8. Quintner JL, Bove G. From neuralgia to peripheral neuropathic pain: evolution of a concept. Regional anesth pain med 2001; 26(4): 368-372. 
9. Hall T, Quintner J. Responses to mechanical stimulation of the upper limb in painful cervical radiculopathy. Australian j physiother. 1996;42(4):277.

10. Sterling M, Treleaven J, Edwards S, Jull G. Pressure pain thresholds of upper limb peripheral nerve trunks in asymptomatic subjects. Physiother res int 2000;5(4):220-9. 11. Walsh J, Kenneally M, Hall T. Pressure pain thresholds of the sciatic, tibial and common peroneal nerves in asymptomatic subjects. Physiotherapy Practice and Research. 2010;31(1):23-7.

12. Vroomen PCAJ, de Krom MCTFM, Knottnerus JA. Consistency of history taking and physical examination in patients with suspected lumbar nerve root involvement. Spine. 2000; 25: 91-7.

13. Smart KM, Curley A, Blake C, Staines A, Doody C. The reliability of clinical judgments and criteria associated with mechanisms-based classifications of pain in patients with low back pain disorders: a preliminary reliability study. Journal of Manual and Manipulative Therapy. 2010;18(2):102-10.

14. Graven-Nielsen T, Wodehouse T, Langford R, Arendt-Nielsen L, Kidd B.

Normalization of widespread hyperesthesia and facilitated spatial summation of deeptissue pain in knee osteoarthritis patients after knee replacement. Arthritis rheum. 2012;64(9):2907-16.

15. Netter FH. Atlas of Human Anatomy. $4^{\text {th }}$ ed. Philadelphia: Saunders/Elservier; 2006. p. $536-537$

16. Lucas NP, Macaskill P, Irwig L, Bogduk N. The development of a quality appraisal tool for studies of diagnostic reliability (QAREL). Journal clin epidemiol. 2010;63(8):854-61. 
17. Vloka JD, Had A, Drobnik L, Ernest A, Reiss W, Thys DM. Anatomical landmarks for femoral nerve block: a comparison of four needle insertion sites. Anesthesia \& Analgesia. 1999;89(6):1467.

18. Field D, Hutchinson JSO. Field's anatomy, palpation and surface markings: Butterworth-Heinemann Medical; 2006. p. 142-143

19. Skou ST, Graven-Nielsen T, Lengsoe L, Simonsen O, Laursen MB, Arendt-Nielsen L. Relating clinical measures of pain with experimentally assessed pain mechanisms in patients with knee osteoarthritis. Scandinavian Journal of Pain. 2013;4(2):111-7. 20. Chesterton, L. S., J. Sim, et al. . Interrater reliability of algometry in measuring pressure pain thresholds in healthy humans, using multiple raters. Clin J Pain 2007 23(9): 760-766.

21. Landis JR, Koch GG. The measurement of observer agreement for categorical data. Biometrics. 1977:159-74.

22. Sim J, Wright CC. The kappa statistic in reliability studies: use, interpretation, and sample size requirements. Phys ther. 2005;85(3):257-68.

23. Shrout PE, Fleiss JL. Intraclass correlations: uses in assessing rater reliability. Psychol Bull. 1979;86(2):420-8.

24. Learmonth YC, Dlugonski DD, Pilutti LA, Sandroff BM, Motl RW. The reliability, precision and clinically meaningful change of walking assessments in multiple sclerosis. Mult Scler. 2013

25. Drouin J. How should we determine a measurement is appropriate for clinical practice. Athl Ther Today. 2003;8(4):2-4. 
26. Atkinson G, Nevill AM. Statistical methods for assessing measurement error (reliability) in variables relevant to sports medicine. Sports medicine. 1998;26(4): 21738.

27. Bland DG, Altman JM. Statistical methods for assessing agreement between two clinical assessments. Lancet. 1986; i: 307-310

28. Stubhaug A. Pressure pain algometry-A call for standardisation of methods. Scandinavian Journal of Pain. 2012;3(1):30.

29. Walton D, Macdermid J, Nielson W, Teasell R, Chiasson M, Brown L. Reliability, standard error, and minimum detectable change of clinical pressure pain threshold testing in people with and without acute neck pain. J orthop sports phys ther. 2011;41(9):644.

30. Palmeira CC, Ashmawi HA, Posso Ide P. Sex and pain perception and analgesia. Rev Bras Anestesiol. 2011; 61(6):814-28.

31. Racine M, Tousignant-Laflamme Y, Kloda LA, Dion D, Dupuis G, Choinière M. A systematic literature review of 10 years of research on sex/gender and experimental pain perception - part 1: are there really differences between women and men? Pain. 2012; 153(3):602-18.

32. Riley JL, Robinson ME, Wise EA Myers CD, Fillingim RB. Sex differences in the perception of noxious experimental stimuli: a meta-analysis. Pain 1998, 74(2-3):181187

33. Fernandez-Carnero J, Fernandez-de-las-Penas C, Sterling M, Souvlis T, ArendtNielsen L,Vicenzino B. Exploration of the Extent of Somato-Sensory Impairment in Patients with Unilateral Lateral Epicondylalgia. J pain, 10, 11 2009:1179-1185 
34. De-la-Llave-Rincon AI, Ortega-Santiago R, Ambite-Quesada S, Gil-Crujera A, Puentedura EJ, Valenza MC, et al. Response of pain intensity to soft tissue mobilization and neurodynamic technique: a series of 18 patients with chronic carpal tunnel syndrome. J Manipulative Physiol Ther. 2012; 35(6):420-7 


\section{TABLES}

Table 1

Pressure Algometry: Intra-rater reliability

\begin{tabular}{llllcl}
\hline Site & $\begin{array}{l}\text { Rater 1A } \\
\text { Mean (SD }\end{array}$ & $\begin{array}{l}\text { Rater 1B } \\
\text { Mean (SD) }\end{array}$ & $\begin{array}{l}\text { ICC (95\% } \\
\left.\mathbf{C I}^{2}\right)\end{array}$ & SEM & $\begin{array}{l}\text { Mean } \\
\text { Diff.(95\% CI) }\end{array}$ \\
\hline Femoral & $168(51)$ & $163(41)$ & $0.69(.41, .84)$ & 26 & $5(-16,26)$ \\
Peroneal & $125(41)$ & $114(37)$ & $0.84(.69, .91)$ & 16 & $11(-7,29)$ \\
Tibial & $141(45)$ & $140(38)$ & $0.64(.32, .81)$ & 25 & $1(-18,20)$ \\
Sciatic & $244(89)$ & $233(82)$ & $0.90(.81, .95)$ & 27 & $11(-28,50)$ \\
\hline
\end{tabular}

standard deviation; ${ }^{2}$ confidence interval

Table 2

Pressure Algometry: Inter-rater reliability

\begin{tabular}{llllcl}
\hline Site & $\begin{array}{l}\text { Rater 1 Mean } \\
\left.\mathbf{( S D}^{1}\right)\end{array}$ & $\begin{array}{l}\text { Rater 2 Mean } \\
(\mathbf{S D})\end{array}$ & $\begin{array}{l}\text { ICC (95\% } \\
\left.\mathbf{C I}^{2}\right)\end{array}$ & SEM & $\begin{array}{l}\text { Mean } \\
\text { Diff.(95\% CI) }\end{array}$ \\
\hline Femoral & $158(55)$ & $176(53)$ & $0.82(.65, .9)$ & 23 & $-17(-41,7)$ \\
Peroneal & $127(38)$ & $136(35)$ & $0.70(.43, .84)$ & 20 & $-10(-26,7)$ \\
Tibial & $136(35)$ & $124(34)$ & $0.56(.16, .77)$ & 23 & $12(-3,28)$ \\
Sciatic & $244(86)$ & $256(69)$ & $0.75(.53, .87)$ & 39 & $-13(-48,23)$ \\
\hline \multicolumn{1}{l}{ standard deviation; ${ }^{2}$ confidence interval } & & & &
\end{tabular}

Table 3

Manual Palpation: Intra-rater reliability

\begin{tabular}{lcc} 
Site & Kappa & $\mathbf{S E}^{\mathbf{1}}$ \\
\hline Femoral & 0.59 & 0.17 \\
Peroneal & 0.55 & 0.15 \\
Tibial & 0.42 & 0.18 \\
Sciatic & 0.60 & 0.14 \\
\hline${ }^{1}$ standard error & &
\end{tabular}

Table 4

Manual Palpation: Inter-rater reliability

\begin{tabular}{lcl}
\hline Site & Kappa & SE $^{\mathbf{1}}$ \\
\hline Femoral & 0.30 & 0.13 \\
Peroneal & 0.49 & 0.15 \\
Tibial & 0.37 & 0.18 \\
Sciatic & 0.60 & 0.13 \\
\hline
\end{tabular}

${ }^{1}$ standard error 
Table 5

Pressure Pain Thresholds

Males vs. Females

\begin{tabular}{lllllll}
\hline Site & Side & $\begin{array}{l}\text { Males } \\
\text { Mean } \\
\text { SD }^{\mathbf{1}} \text { ) }\end{array}$ & $\begin{array}{l}\text { Females } \\
\text { Mean (SD) }\end{array}$ & Mean Diff. & $\mathbf{9 5 \% \mathbf { C I } ^ { \mathbf { 2 } }}$ & P value \\
\hline Femoral & $\mathrm{L}$ & $187(58)$ & $148(42)$ & 39 & $(16,61)$ & 0.001 \\
& $\mathrm{R}$ & $181(50)$ & $150(36)$ & 31 & $(11,51)$ & 0.002 \\
\multirow{2}{*}{ Peroneal } & $\mathrm{L}$ & $139(36)$ & $125(36)$ & 14 & $(-2,31)$ & 0.07 \\
& $\mathrm{R}$ & $120(35)$ & $119(43)$ & 0.83 & $(-17,19)$ & 0.93 \\
\multirow{2}{*}{ Tibial } & $\mathrm{L}$ & $148(29)$ & $113(32)$ & 35 & $(22,49)$ & 0 \\
& $\mathrm{R}$ & $162(36)$ & $121(37)$ & 41 & $(25,57)$ & 0 \\
Sciatic & $\mathrm{L}$ & $290(74)$ & $212(61)$ & 77 & $(47,108)$ & 0 \\
& $\mathrm{R}$ & $208(85)$ & $199(65)$ & 81 & $(47,115)$ & 0
\end{tabular}

standard deviation; ${ }^{2}$ confidence interval

Table 6

Pressure Pain Thresholds

Left vs. Right

\begin{tabular}{llllll} 
Site & Left Mean (SD & Right Mean (SD) & Mean Diff. & $\mathbf{9 5 \%} \mathbf{C I}^{\mathbf{2}}$ & P value $^{\mathbf{1}}$ \\
\hline Femoral & $167(54)$ & $165(46)$ & 2 & $(-8,11)$ & 0.73 \\
Peroneal & $132(37)$ & $120(39)$ & 12 & $(4,20)$ & 0.003 \\
Tibial & $130(35)$ & $141(42)$ & -11 & $(-19,-3)$ & 0.007 \\
Sciatic & $250(78)$ & $238(85)$ & 12 & $(-3,26)$ & 0.12 \\
\hline
\end{tabular}

${ }^{1}$ standard deviation; ${ }^{2}$ confidence interval 
Fig 1: Manual palpation at the A) femoral, B) sciatic, C) tibial and D) common peroneal nerves

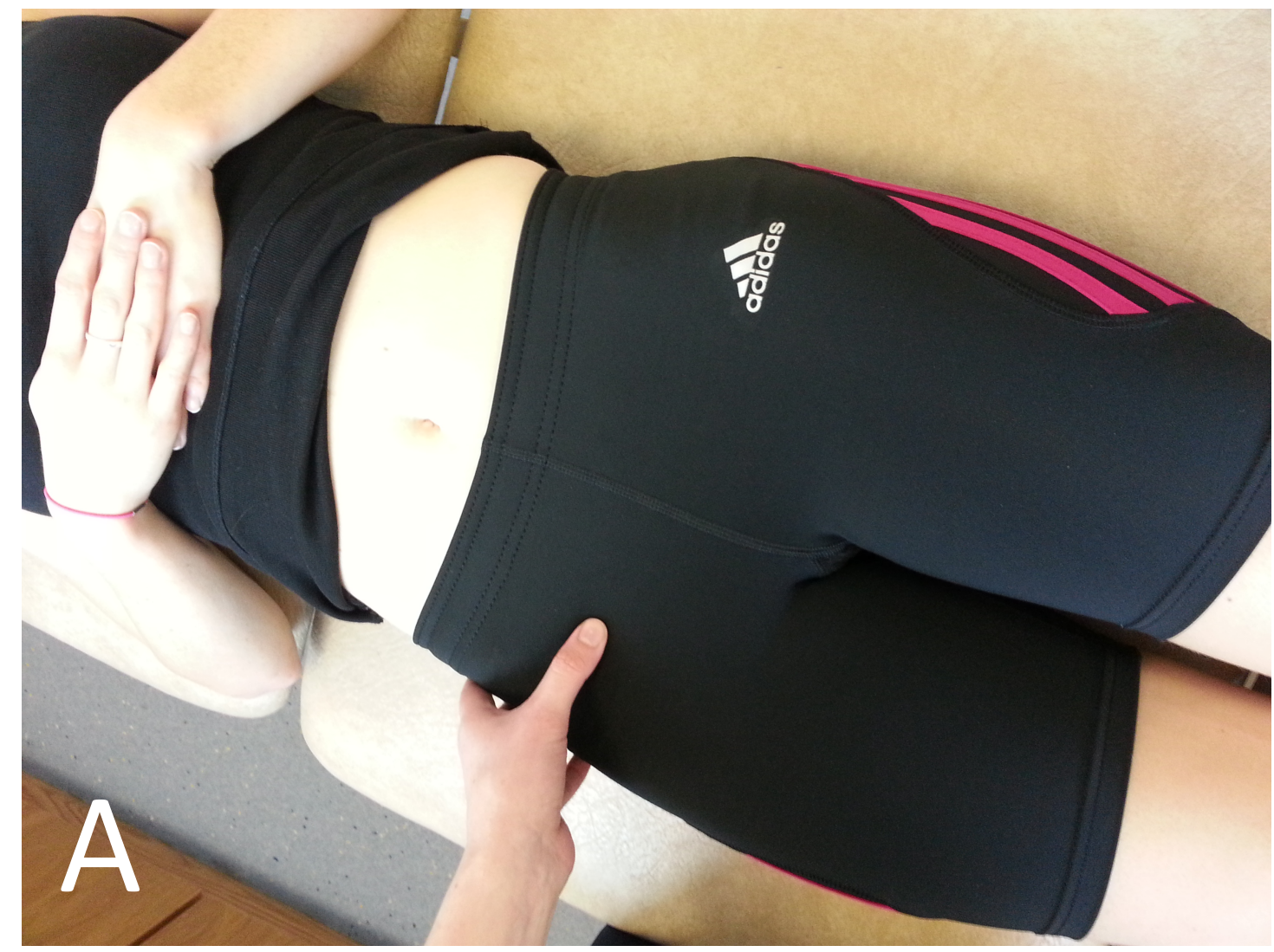



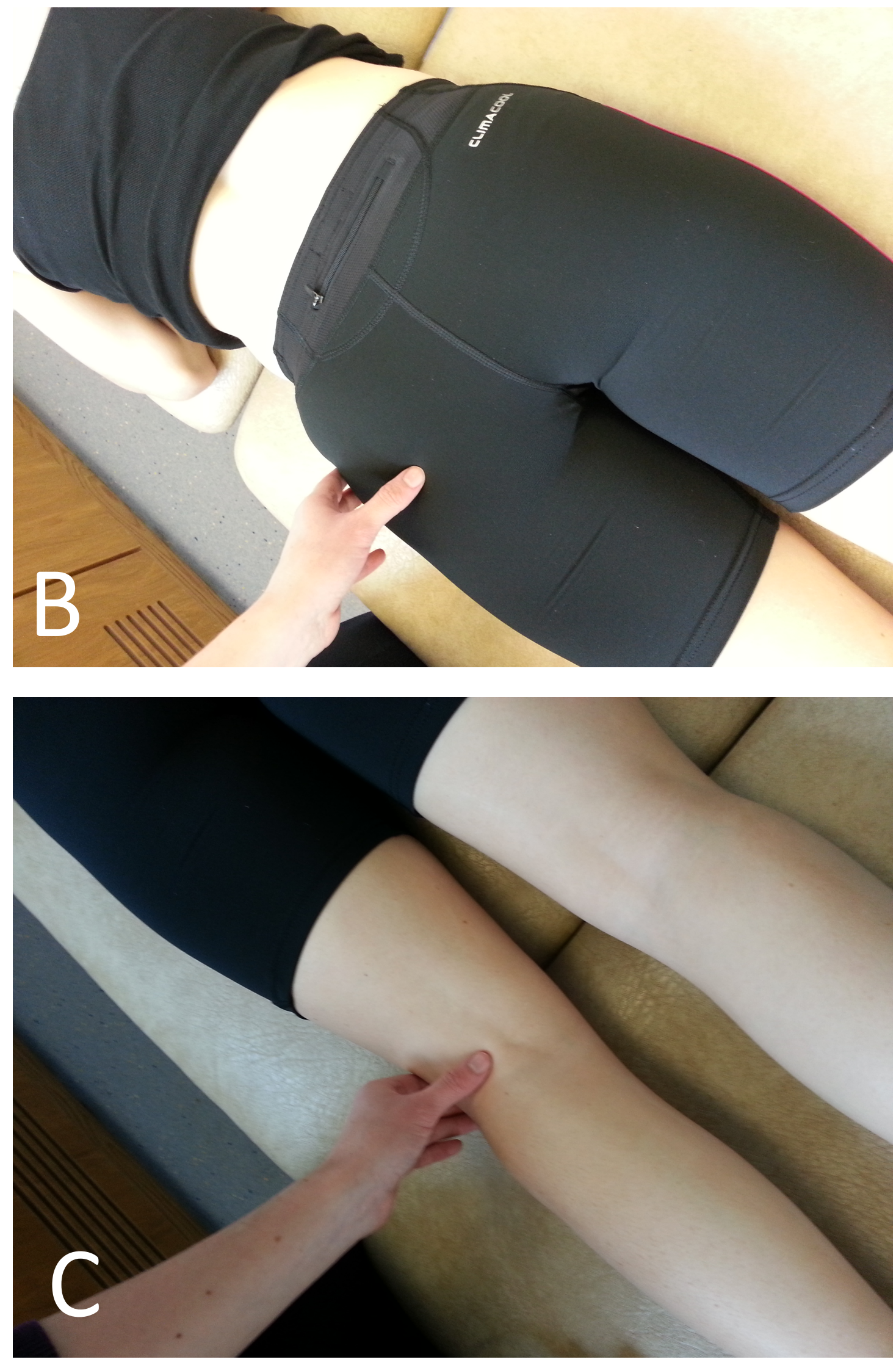


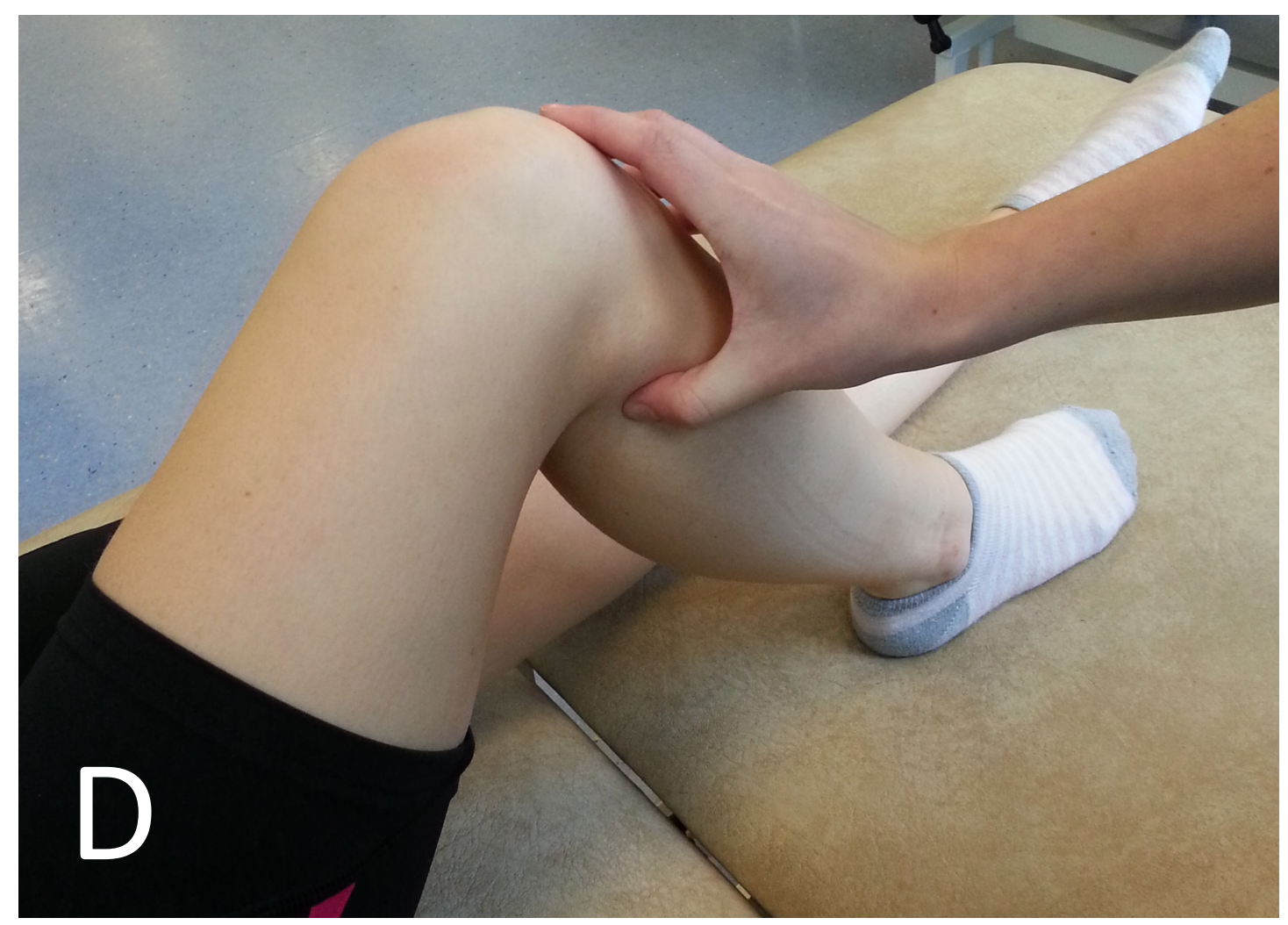


Fig 2: Pressure algometry at the A) femoral, B) sciatic, C) tibial and D) common peroneal nerves

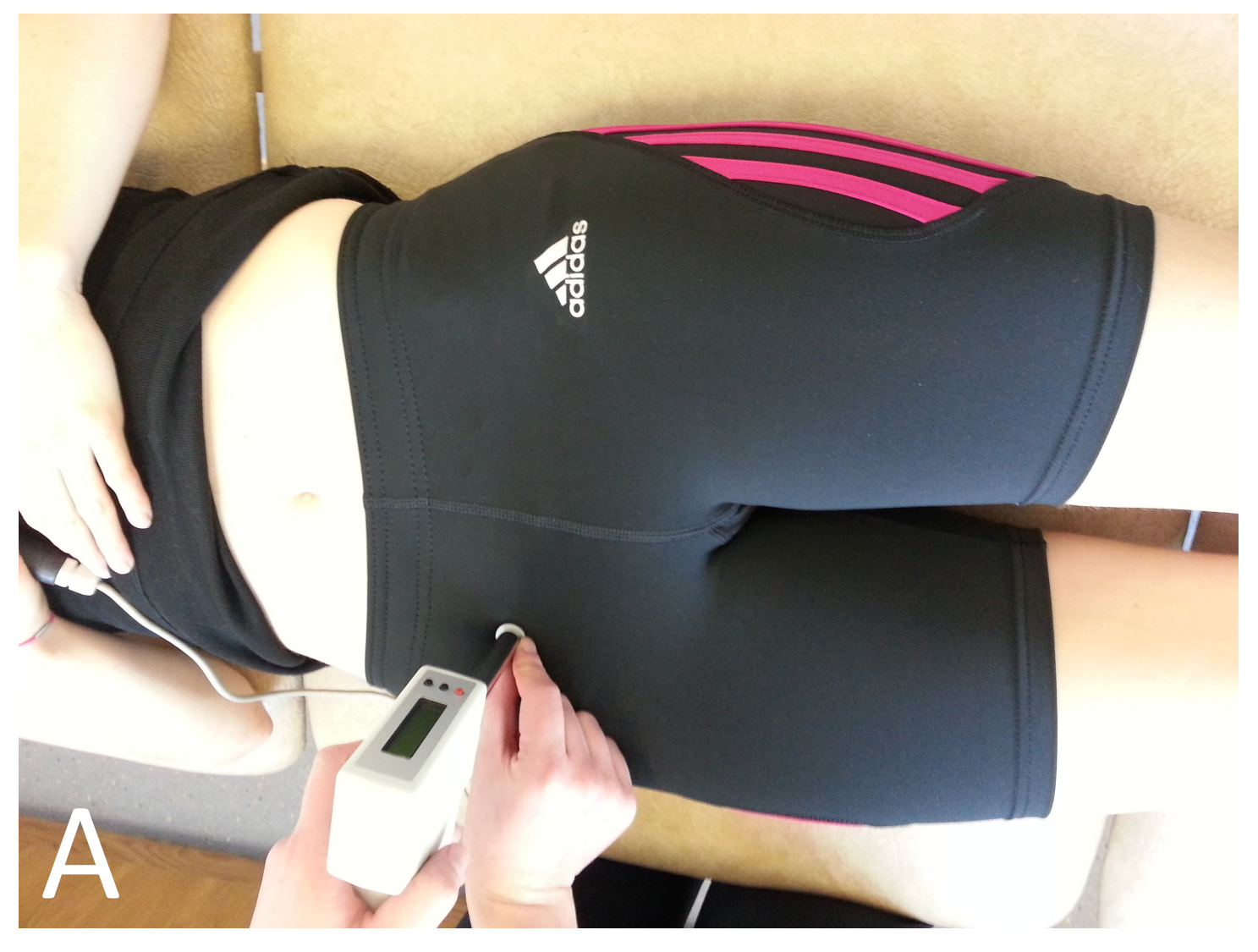



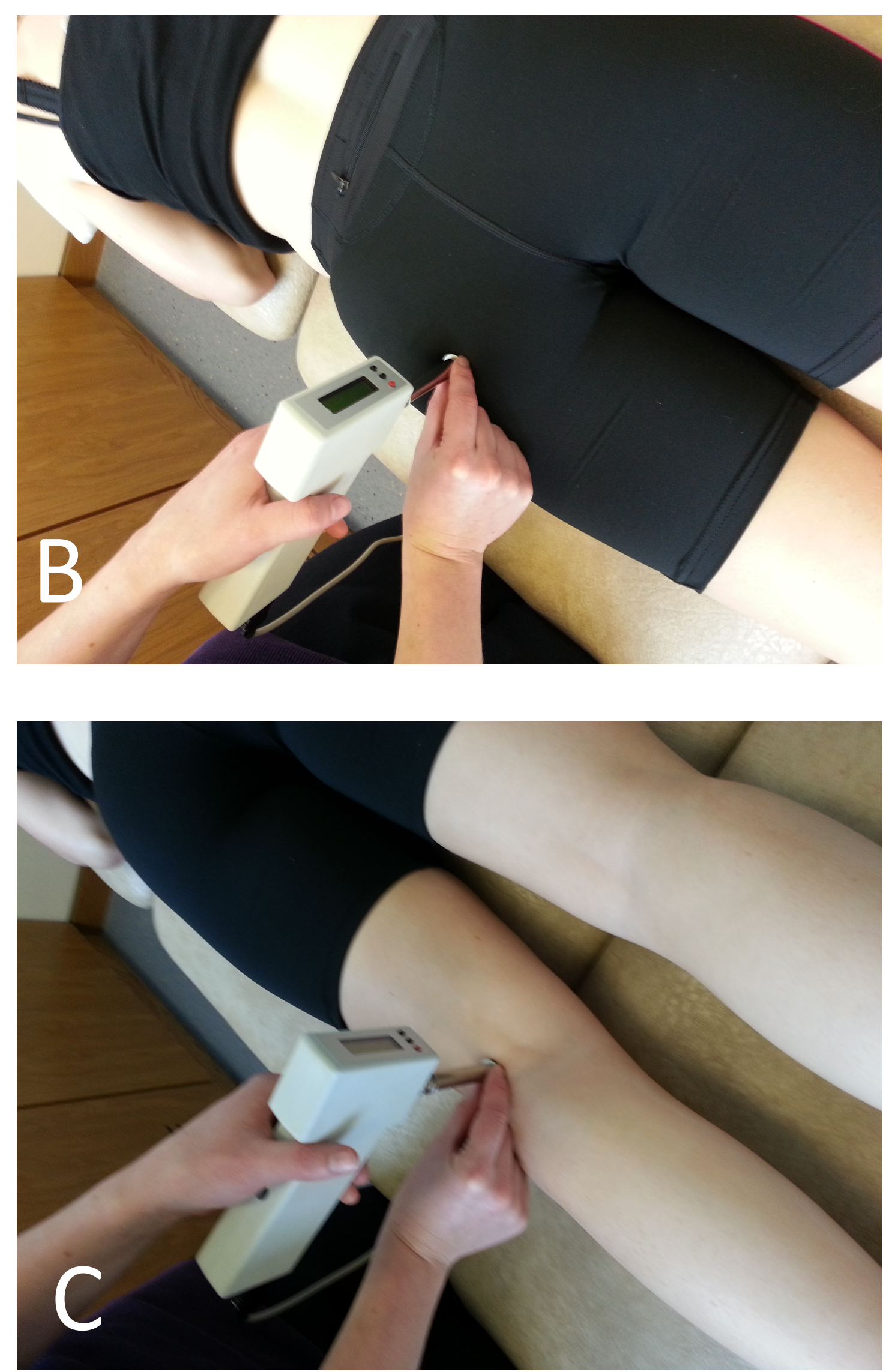


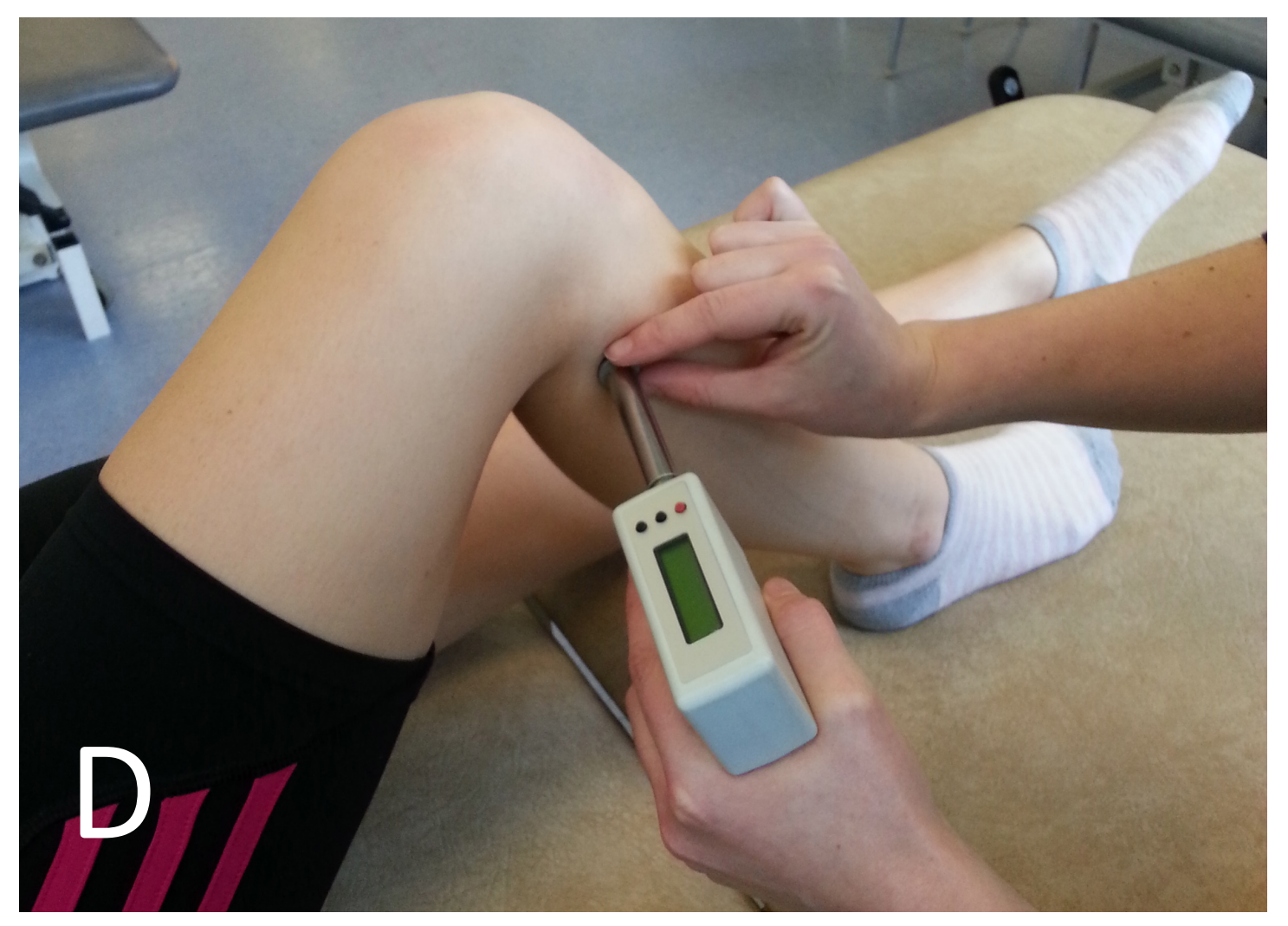




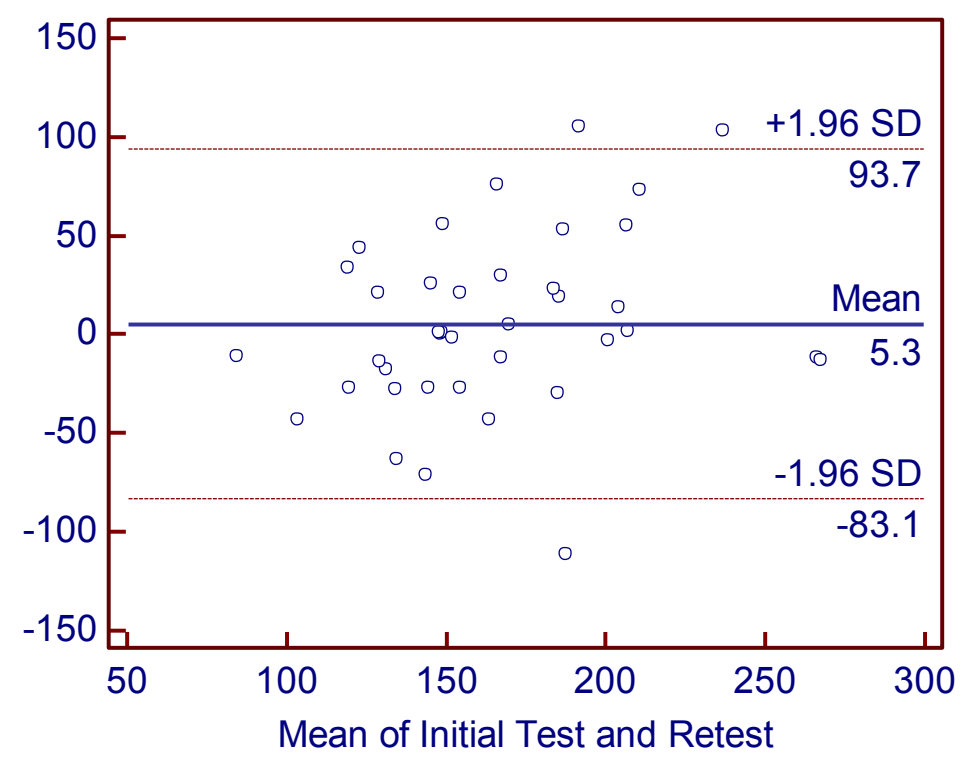

Fig. 3. Bland Altman Plot: Femoral nerve intra-rater reliability

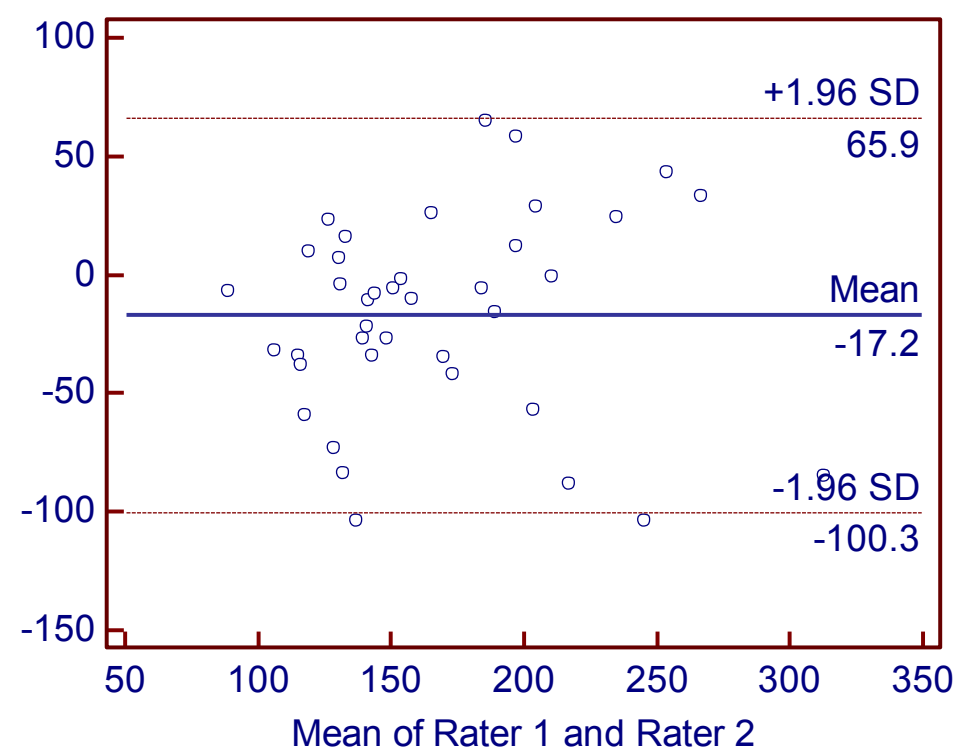

Fig. 4. Bland Altman Plot: Femoral nerve inter-rater reliability 